\title{
An Analog FSCV Device Combined with a Phase-Locked Loop for in Vivo Detection of Dopamine
}

\author{
Muhammad Haziq, Raheel Riaz, Samet Kocatürk, Ahsan Ayyaz, Mehmet Kocatürk*
}

\begin{abstract}
Objective: In this work, our goal was to develop an easy-to-implement FSCV device which can be reliably employed for in vivo detection of dopamine. Methods: Readily available analog integrated circuits (ICs) were used to generate monopolar triangular voltammetric waveforms with adjustable holding and peak potentials. The timing of the voltammetric scans were controlled by a phase-locked loop implemented around a standard microcontroller so that the power line frequency noise, which generally complicates the detection of dopamine through the recordings, could be effortlessly eliminated by backgroundsubtraction. Results: The practicality of the system was tested in vitro using a dopamine solution and in vivo using a freely moving rat chronically implanted with a carbon fiber microelectrode in the ventromedial striatum (VMS). The system consistently detected dopamine in the solution and dopamine released in the VMS in response to presentation of reward. Conclusion: We demonstrated a low-cost and easily reproducible solution specifically designed for in vivo measurements of dopamine from behaving subjects. Significance: Based on utilization of analog ICs, the triangular waveform generated in the present system is linear in contrast to the conventional, digital-to-analog converterbased systems, which fundamentally approximates a staircase form to a linear ramp. We believe the present technique can be applied for the measurement of other electrochemically active analytes requiring a triangular waveform and elimination of power line frequency noise.
\end{abstract}

Index Terms-Dopamine, electrochemistry, fast-scan cyclic voltammetry, neuromodulator, neuroscience, phase-locked loop.

\section{INTRODUCTION}

$\mathrm{R}^{\mathrm{s}}$ ESEARCH into motor control, goal-directed behavior and reinforcement learning is significantly focused on the characterization of phasic changes in the activity of the

This work was supported by The Scientific and Technological Research Council of Turkey (TÜBİTAK), Grant No: EEEAG-117E286 and SBAG$118 \mathrm{~S} 072$.

M. Haziq and A. Ayyaz are with Department of Biomedical Engineering, Istanbul Medipol University, Istanbul, Turkey.

R. Riaz was with Department of Biomedical Engineering, Istanbul Medipol University, Istanbul, Turkey. He is now with Faculty of Science and Technology, Free University of Bozen-Bolzano, Bozen-Bolzano, Italy.

S. Kocatürk was with Department of Biomedical Engineering, Istanbul Medipol University, Istanbul, Turkey. He is now with Center for Molecular and Behavioral Neuroscience, Rutgers University-Newark, Newark, NJ, USA.

*M. Kocatürk is with Health Sciences and Technology Research Institute, Department of Biomedical Engineering, Istanbul Medipol University, Istanbul, Turkey (correspondence e-mail: mkocaturk@ medipol.edu.tr). midbrain dopaminergic neurons and the modulations of dopamine concentration in the structures innervated by dopaminergic axons [1]-[3]. Electrophysiology enables recording the activity of dopaminergic neurons with high temporal and spatial resolution [4], [5]. In addition to electrophysiology, fast-scan cyclic voltammetry (FSCV), as another powerful technique, offers the capability of monitoring the phasic dopamine release in the striatal structures with high temporal resolution and spatial acuity [6][9].

FSCV, as an electroanalytical technique, is used to measure the concentration of electrochemically active molecules in the vicinity of an electrode [10]. It applies electrical potential to the electrode and induces oxidation and reduction of the analyte at the surface of the electrode. These reactions lead to faradaic current flow between the electrode and the analyte. This current is directly proportional to the concentration of the analyte in the medium [11]. In the case of in vivo detection of dopamine via FSCV, carbon fiber microelectrodes are conventionally used due to their small size, biocompatibility and inertness during application of voltage scans [12]-[14]. It is implanted into the target tissue and a triangular voltammetric waveform is applied to the microelectrode versus a $\mathrm{Ag} / \mathrm{AgCl}$ reference electrode [15]. Typically, the applied potential is held at $-0.4 \mathrm{~V}$ versus the reference electrode. During the scans it is linearly ramped to a maximum voltage of $1.3 \mathrm{~V}$ at a scan rate of $400 \mathrm{~V} / \mathrm{s}$ and returns to the holding potential $(-0.4 \mathrm{~V})$ with the same scan rate [16]-[19]. The voltammetric scan is, therefore, completed within $8.5 \mathrm{~ms}$. The faradaic currents occurring during the scans are then used to detect the concentration of dopamine. In order to extract the faradaic currents through the scans, cyclic voltammograms (CVs), which present the overall current passing through electrode versus applied voltage, are collected for a baseline period and these $\mathrm{CVs}$ are then subtracted from the ones acquired in following scans. The remaining current in the subsequent scans after subtraction is faradaic current, in other words background-subtracted current, which represents the change in the concentration of dopamine in the vicinity of the implanted microelectrode [20], [21]. Therefore, FSCV allows monitoring the phasic changes in dopamine concentration relative to a baseline reference point [22]-[24].

The conventional approach for detection of dopamine (and other electrochemically active analytes) via FSCV has been 
based on employing an operational amplifier (opamp) in $\mathrm{I} / \mathrm{E}$ converter mode: The working electrode is directly connected to the inverting input of the operational amplifier and the voltammetric waveform is applied to its non-inverting input. In this configuration, the voltage of the working electrode follows the waveform voltage and the resulting current passing through the electrode is converted to voltage at the output of the operational amplifier [25]. The voltage output of the operational amplifier is recorded using a PC or another digital system and the current passing through the electrode is afterwards extracted from the recordings to create CVs.

Since the faradaic currents generated by redox reactions of physiological levels of dopamine are in the orders of nanoamperes [26]-[28], the development of a reliable and low-noise FSCV system which supports in vivo detection of dopamine is a non-trivial problem. There are commercially available professional devices such as WaveNeuro Potentiostat System and WaveNow Wireless Potentiostat System (Pine Research Instrumentation, NC, USA) and research devices in the literature which allows dopamine measurement using FSCV technique [29]-[31]. In these FSCV devices, the voltammetric waveform is generated using a digitial-to-analog converter (DAC). Instead of using an integrated FSCV device, other solution for detection of dopamine is to create a FSCV setup where the triangular waveform is produced by a data acquisition device controlled by a PC and applied to the working electrode through a I/E converter [32], [33]. In such setting, data acquisition card also employs a DAC fundamentally for the generation of the waveform. In order to create a waveform with a high scan rate (e.g. $400 \mathrm{~V} / \mathrm{s}$ ) and close to a true triangle, use of a DAC with a high resolution and a high update rate is necessary. In addition, powerful microprocessors and advanced embedded system programming skills are required to control these integrated circuits (ICs). These requirements significantly increases the development costs of the equipments used in FSCV.

In the present article, we propose a novel FSCV device which generates triangular voltammetric waveform without using DAC and is specifically designed for facilitating in vivo detection of dopamine. In lieu of employing a DAC, the present device implements a customized analog circuit which converts pulses generated by a standard microcontroller into triangular waves. Triangular waves are followed by a DC holding potential and repeated every $100 \mathrm{~ms}$ for online monitoring of dopamine transients as in conventional DACbased systems. The present design approach decreases the development and implementation costs of FSCV systems which use triangular scans, and allows easy implementation in a standard neuroscience research laboratory using readily available electronic components. The present design based on analog circuit components also enables generation of true triangular waves with linear, continuous rising and falling ramps in contrast to the existing systems, which basically generate a staircase waveform due to use of DAC. In addition, the holding and peak voltages, and the scan rate for the anodic and cathodic sweep can be easily adjusted using the present device. Here we describe the structure of the proposed FSCV device combined with a low-cost phase-locked loop (PLL) circuit we implemented to eliminate the power line noise in the recordings, and validate the practicality of the device by in vivo measurements acquired from a freely moving rat chronically implanted with a carbon fiber microelectrode. We also show how to interface the device with a desktop PC equipped with a data acquisition (DAQ) card and a commutator allowing continuous recordings from a tethered rat.

\section{METHODS}

\section{A. Structure of the FSCV Hardware}

The FSCV hardware presented here was designed to enable measurement of dopamine from freely moving subjects. Fig. 1 illustrates the FSCV hardware and its interfacing with an experimental setup, and Fig. 2 demonstrates the circuit diagram of the hardware. The FSCV hardware was composed of four modules: 1) waveform generator 2) phase-locked loop (PLL), 3) headstage and 4) amplifier. In this section, we describe the design and implementation of these modules.

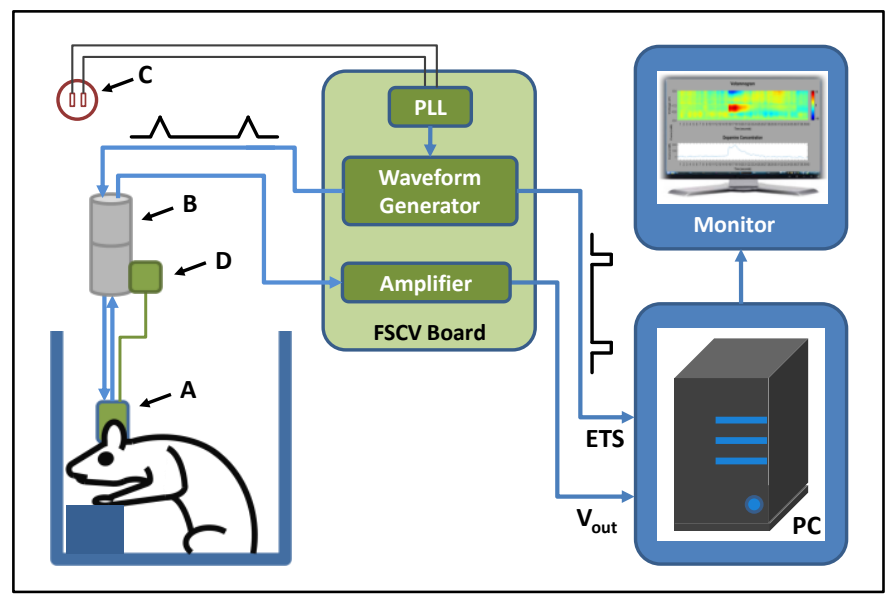

Fig. 1. Block diagram of the FSCV hardware and the experimental setup. The headstage (A) implementing a $\mathrm{I} / \mathrm{E}$ converter was fixed on rat's head and received triangular signal from the waveform generator through a commutator (B) used to keep the headstage cables untangled during recordings. The output of the headstage was fed to the amplifier through the commutator and the voltammetric signals $\left(\mathrm{V}_{\text {out }}\right)$ were recorded using a PC equipped with a data acquisition card. A phase-locked loop (PLL) was used to match the phase of the generated triangular waveform to the phase of mains (C). The pulses indicating the starting times of the scans were used as the external trigger signal (ETS) to initiate the data acquisition every $100 \mathrm{~ms}$. Batteries (D) mounted onto the commutator directly supplied power for the headstage.

\section{1) Waveform generator}

The waveform generator module of the FSCV hardware was used to produce the triangular voltammetric waveform which was applied to a working microelectrode versus a reference electrode for detection of dopamine. Fig. 2B shows the circuit diagram of the waveform generator. The waveform generator basically consisted of analog circuit elements and converted pulses with a width of $4.25 \mathrm{~ms}$ into triangular waves with a width of $8.5 \mathrm{~ms}$ using an integrator circuit. The first stage of the waveform generator was a summing amplifier and used to offset the pulse signal fed to the second, integration stage. The 


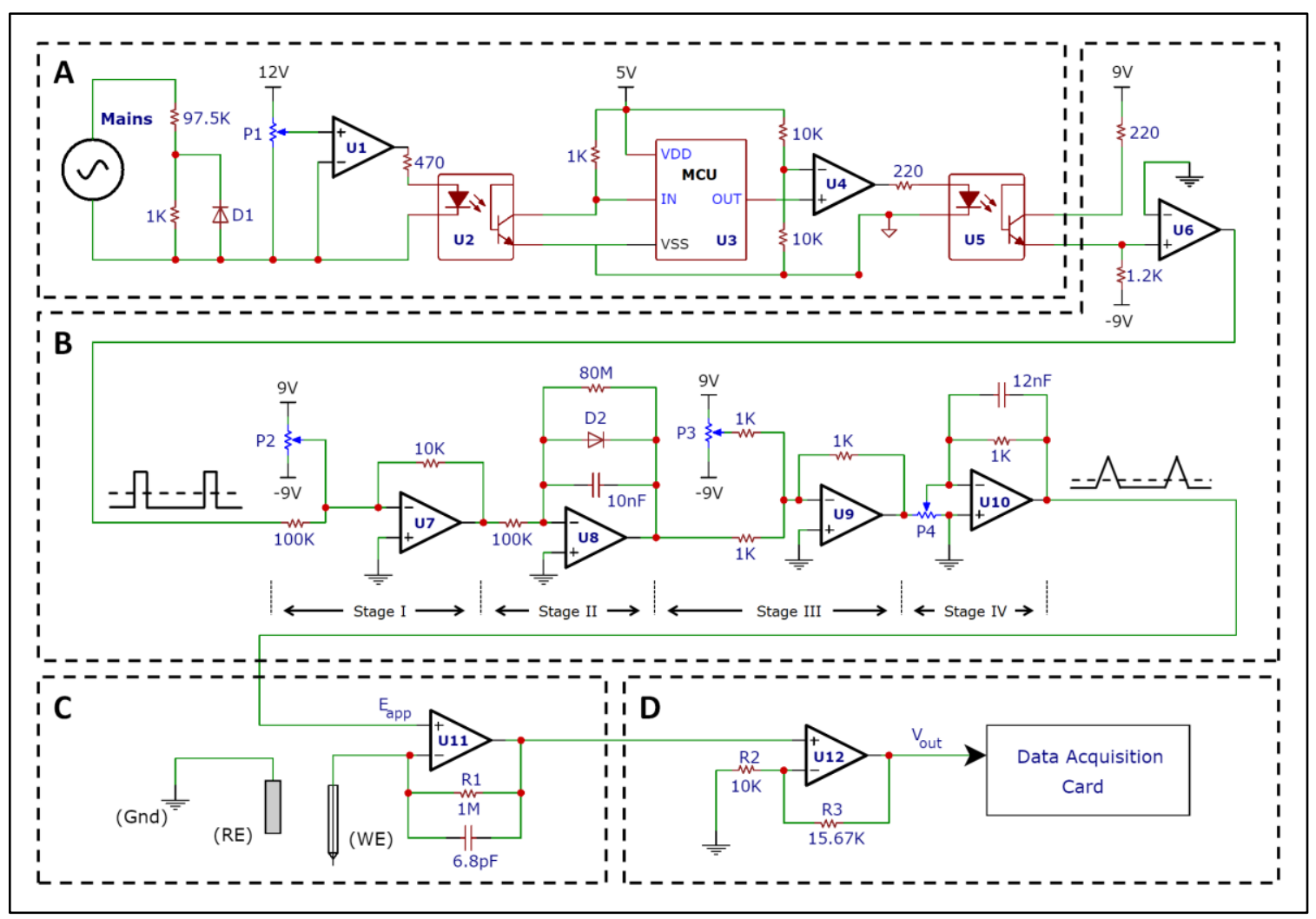

Fig. 2. Circuit diagram for the FSCV hardware. (A) Phase-locked loop (PLL) module. MCU: microcontroller unit. (B) Waveform generator module. (C) Headstage module. RE: reference electrode, WE: working electrode, Gnd: ground. (D) Amplifier module.

offsetting process at this stage using potentiometer P2 was applied to create symmetry between the maximum and minimum voltages of the pulse signal at the input of the integration stage. This symmetry in the pulse signal was required for obtaining symmetry between the rising and falling slopes of the triangular waveform at the output of the integration stage. The offset and amplitude of triangular waveform generated by the integration stage was then adjusted by potentiometers $\mathrm{P} 3$ and $\mathrm{P} 4$, respectively, in the third and fourth stages. Diode D2 (1N4005) was utilized within the integration stage to prevent the operational amplifier U8 from saturation and create a rectified, monopolar triangular waveform. The operational amplifiers used for U7 and U8 were TL07x series (Texas Instruments, TX, USA) because of their high slew rate $(13 \mathrm{~V} / \mu \mathrm{s})$ and low input bias current (65 $\mathrm{pA}$ ), which are required for offsetting the pulse signal at the first stage and limiting the DC gain of U8 at the integration stage with a large-value feedback resistor (i.e. $80 \mathrm{M} \Omega$ ), respectively [34]. The operational amplifiers used for U8 and U9 were also TL07x series due to their easy availability and low-cost. The voltage comparator used for U6 is LM311 (Texas Instruments) because of their fast response time (i.e. $165 \mathrm{~ns})$ and wide supply voltage range.

\section{2) Phase-locked loop}

Capacitive coupling between the power line and the tissue is a significant source of noise, which hampers the detection of the small faradaic currents resulting from the redox reactions of physiological levels of dopamine. This type of noise can be eliminated by subtracting the background current if the phases of the mains signal and the applied voltammetric waveform precisely match each other [35]. For monitoring the line frequency and controlling the timing of the voltammetric waveform, we implemented the PLL module of the FSCV device presented here (see Fig. 2A). In the PLL module, the mains voltage was divided by approximately 100 using $1 \mathrm{~W}$ resistors, half-wave rectified by a diode (D1, 1N4007) and converted into pulses using a comparator (U1). The generated pulse signal was continuously monitored by a microcontroller (U3) to detect the mains phase and output phase-locked pulses with a width of $4.25 \mathrm{~ms}$, which were later converted into triangle waves via the waveform generator module. The width of the pulses was precisely controlled using timer of the microcontroller. The frequency of the phase-locked pulses produced by the microcontroller was $10 \mathrm{~Hz}$. The duty cycle of the pulses at the input of the microcontroller was $65 \%$, set by potentiometer P1 in the PLL module. Mains voltage was optically isolated from the microcontroller using an optocoupler (U2) for electrical safety. Another optocoupler (U5) was used between the PLL and waveform generator modules for isolating their power supplies from each other. The optocouplers used for U2 and U5 were 4N35 (Vishay Semiconductors, PA, USA) and the microcontroller used for U3 was PIC18F4520 (Microchip Technology, AZ, USA).

\section{3) Headstage and amplifier}

The headstage (see Fig. 1) was used to apply the triangular waveform to the working microelectrode versus the $\mathrm{Ag} / \mathrm{AgCl}$ reference electrode. Fig. 2C illustrates the circuit diagram of 
the headstage, implementing a I/E converter using an operational amplifier [25]. TLC227x series (Texas Instruments) operational amplifier was used for U11 due to their low input bias current (1 pA), low input current noise $(0.6 \mathrm{fA} / \sqrt{ } \mathrm{Hz})$ and availability in small size with quad-channel versions (e.g. TLC2274IPW), which could be advantageous for future applications requiring a higher number of recording microelectrodes. The power for headstage was directly supplied from separate batteries (see Fig. 1) instead of providing it through the commutator so that any significant noise in the supply voltage of the headstage that could be caused by the rotation of the commutator was avoided. Lithium-ion batteries $(3.7 \mathrm{~V})$ were used to supply voltage to the headstage due to their light-weight. In the headstage, an operational amplifier with low input bias current and high input impedance was used (i.e. TLC227x series) to prevent any large distortion in the voltammetric waveform received from the FSCV board through the commutator (see Fig. 1 and Fig. 2). Three channels of a 32-channel commutator (Plexon Inc., TX, USA) was used in the recording. One channel was used for the connection between the ground and the reference electrode, another one was used for transmitting voltammetric waveform to the headstage and the other was used for transmitting the output of the headstage (see Fig. 2C)

The output of the headstage was fed to the amplifier module of the FSCV hardware (see Fig. 1). The amplifier module was used to adjust the maximum amplitude of the signal at the input of the data acquisition card inserted into the PC so that the full voltage range of its analog input channel could be used while avoiding saturation. Typically, with chronically implanted carbon fiber microelectrodes we set the gain of the amplifier to 2.567 as illustrated in Fig. 2D. Using the present configuration, the current from the working microelectrode $(i)$ could be calculated by measuring the voltage at the output of the FSCV $\left(V_{\text {out }}\right)$ device as follows:

$$
i=\frac{V_{\text {out }} R_{2}}{R_{1}\left(R_{2}+R_{3}\right)}-\frac{E_{a p p}}{R_{1}}
$$

where $E_{a p p}$ was the voltage applied to the working electrode or the non-inverting input of the opamp in the headstage (U11), $R_{1}$ was the feedback resistor in the headstage, $R_{2}$ and $R_{3}$ were the resistors to adjust the gain of the amplifier module.

\section{4) Data acquisition}

The output of the voltammetry hardware $\left(V_{\text {out }}\right)$ was recorded using a PC equipped with a data acquisition card (Fig. 1). The DAQ device (National Instruments, PCI-6040E, TX, USA), which supports 12-bit resolution, was configured to perform analog-to-digital conversion (ADC) through one channel with a sampling rate of $200 \mathrm{kHz}$. LabVIEW software (National Instruments) was used to control the DAQ device. In order to phase-lock the recordings to mains, the pulses used for generation of the triangular waveform were also employed for externally triggering the DAQ card (see Fig. 1 and Fig. S1). The DAQ device was configured to acquire 3000 samples for each triggering pulse. 1700 samples, which correspond to the voltammetric scan time $(8.5 \mathrm{~ms})$, were used to construct CVs.

\section{B. Microelectrode and Reference Electrode Fabrication}

Chronically implantable microelectrodes and reference electrodes were fabricated as previously described [6], [36]. A single carbon fiber with a diameter of $7 \mu \mathrm{m}$ (Goodfellow Cambridge Ltd. \#C005722, UK) was aspirated into a polyimide coated fused silica capillary (Polymicro Technologies \#TSP020090, AZ, USA) with an outer diameter of $90 \mu \mathrm{m}$. One end of the capillary was sealed using a twocomponent epoxy (Epoxy Technology Inc. EPO-TEK 301, MA, USA). The epoxy was applied after becoming viscous so that a smooth, bulb-shaped microelectrode tip could be formed to reduce the tissue damage during implantation. The other end of the capillary was fixed onto a copper wire which has a diameter of $1 \mathrm{~mm}$ using epoxy and silver paint (Loctite Co. \#3863, Germany) was applied to provide the electrical connection between the carbon fiber and the wire. The silver paint, after drying, was coated using a thin layer of dental acrylic to further secure the electrical connection between the wire and the carbon fiber. Finally, the carbon fiber protruding from the other end of the capillary was cut to a length of 150$200 \mu \mathrm{m}$ to prepare the recording tip of the microelectrode.

Reference $(\mathrm{Ag} / \mathrm{AgCl})$ electrodes were fabricated via electrolysis. A silver wire with a diameter of $0.2 \mathrm{~mm}$ was used to make the reference electrode. The positive terminal of a $5 \mathrm{~V}$ DC power supply with $500 \mathrm{~mA}$ current limitation was connected to the silver wire and the negative terminal to a stainless steel wire. Both wires immersed into a hydrochloric acid solution (36\%) and the current was applied for approximately 2-3 minutes until the bubbles disappear, indicating that the electrolysis process was complete.

\section{Voltammetry Surgery}

All animal procedures presented in this paper were approved by and conducted in accordance with the regulations of the Istanbul Medipol University Ethics Committee on Animal Maintenance and Experimentation. One Wistar male rat weighing $420 \mathrm{~g}$ was chronically implanted with a carbon fiber microelectrode in the ventromedial striatum (VMS) in the left hemisphere (anteroposterior $+1.5 \mathrm{~mm}$, mediolateral $2.1 \mathrm{~mm}$, dorsoventral $6.4 \mathrm{~mm}$ ) and a $\mathrm{Ag} / \mathrm{AgCl}$ reference electrode in the contralateral hemisphere (anteroposterior +1.5 $\mathrm{mm}$, mediolateral $+2.1 \mathrm{~mm}$, dorsoventral $3.0 \mathrm{~mm}$ ) [37]. Implantation of the electrodes was performed after i.p. injection $(1.5 \mathrm{cc} / \mathrm{kg})$ of a mixture of ketamine and xylazine (100 mg/kg and $12 \mathrm{mg} / \mathrm{kg}$ in saline, respectively). Maintenance of anesthesia was achieved with isoflurane gas as needed. Craniotomy was created bilaterally and the dura mater was removed over the implantation sites carefully while avoiding damaging blood vessels and the pia mater [38]. The carbon fiber microelectrode was lowered into the target position slowly $(100 \mu \mathrm{m} / \mathrm{min})$ after reaching a depth of $6 \mathrm{~mm}$. The positions of the working and reference electrodes were fixed using dental acrylic. A female SubMiniature version A (SMA) connector was fixed onto the dental acrylic on the head of the rat. The copper wire of the carbon fiber microelectrode was soldered to the inner pin and the reference wire to the 
outer pin of the SMA connector. SMA connector was preferred for the connection between the headstage and the implanted electrodes due their screw-type coupling mechanism providing the reliability in the electrical connection (see Fig. S2). The rat was given 1 month to recover from the surgery before initiating the voltammetric recording [13], [32].

\section{RESULTS}

The practicality of the FSCV device presented here was tested by performing measurements of dopamine concentration both in vitro and in vivo. For the in vitro measurements, we recorded the background current from a saline solution and performed another recording from the same solution after addition of dopamine. The dopamine concentration of the latter solution was $1 \mu \mathrm{M}$. Fig. 3A demonstrates the color plot of the background-subtracted voltammetric signal acquired from this solution. The corresponding background-subtracted cyclic voltammogram for a single scan is shown in Fig. 3D.

Secondly, the FSCV device was examined for in vivo detection of dopamine using a rat chronically implanted with a carbon fiber microelectrode in the ventromedial striatum (see Voltammetry Surgery). Prior to the initiation of the recording, the rat was placed into the experimental setup represented in Fig. 1 and tethered to the FSCV device for 1 hour for stabilization of the background current. Fig. 3B displays the background-subtracted voltammetric signal acquired from the rat in response to presentation of reward (i.e. $20 \mu \mathrm{l}$ of $30 \%$ sucrose solution) using a pipette. The changes in redox currents as a function of the applied voltage $\left(E_{a p p}\right)$ are illustrated in the plot by color codes. The reward presentation time is indicated by black triangular marker at the top of the plot. The background-subtracted cyclic voltammogram corresponding to the single scan pointed by the gray triangular marker is presented in Fig. 3E. The output of the FSCV device ( $V_{\text {out }}$ in Fig. 2) for a single scan during the present recording is demonstrated in Fig. 3F.

Finally, we tested the noise in the whole system. In order to evaluate the noise in the system, we connected a resistor between the inverting input of the operation amplifier in the headstage (U11) and ground (see Fig. 2), and performed recording using the data acquisition system presented here (see Data acquisition). The resistor here was employed to simulate the current passing through the working microelectrode. The value of the resistor was $1.2 \mathrm{M} \Omega$ to obtain a peak background current (i.e. $1.026 \mu \mathrm{A}$ ) comparable to the one recorded in vivo (i.e. $1.066 \mu \mathrm{A}$, see Fig. 3F and Eq. (1). Fig. 3C demonstrates the background-subtracted current passing through the resistor as a function of the applied voltage. The background $\mathrm{CV}$ was calculated by averaging all scans included in the present plot. The overall standard deviation of the noise across this recording was $2.913 \mathrm{nA}$ without any filtering and $0.138 \mathrm{nA}$ with centered moving average filtering with a window size of 61 , which was also used in the plot in Fig. 3B. The overall standard deviation of the noise in Fig. 3B within the first three seconds, where the background-subtracted $\mathrm{CVs}$ were stabilized, was $2.836 \mathrm{nA}$ without any filtering and $0.313 \mathrm{nA}$ with centered moving average filtering with a window size of 61. Fig. 3G demonstrates the triangular waveform generated by the present FSCV device. The present triangular waveform was recorded by the data acquisition system described here (see Data acquisition).

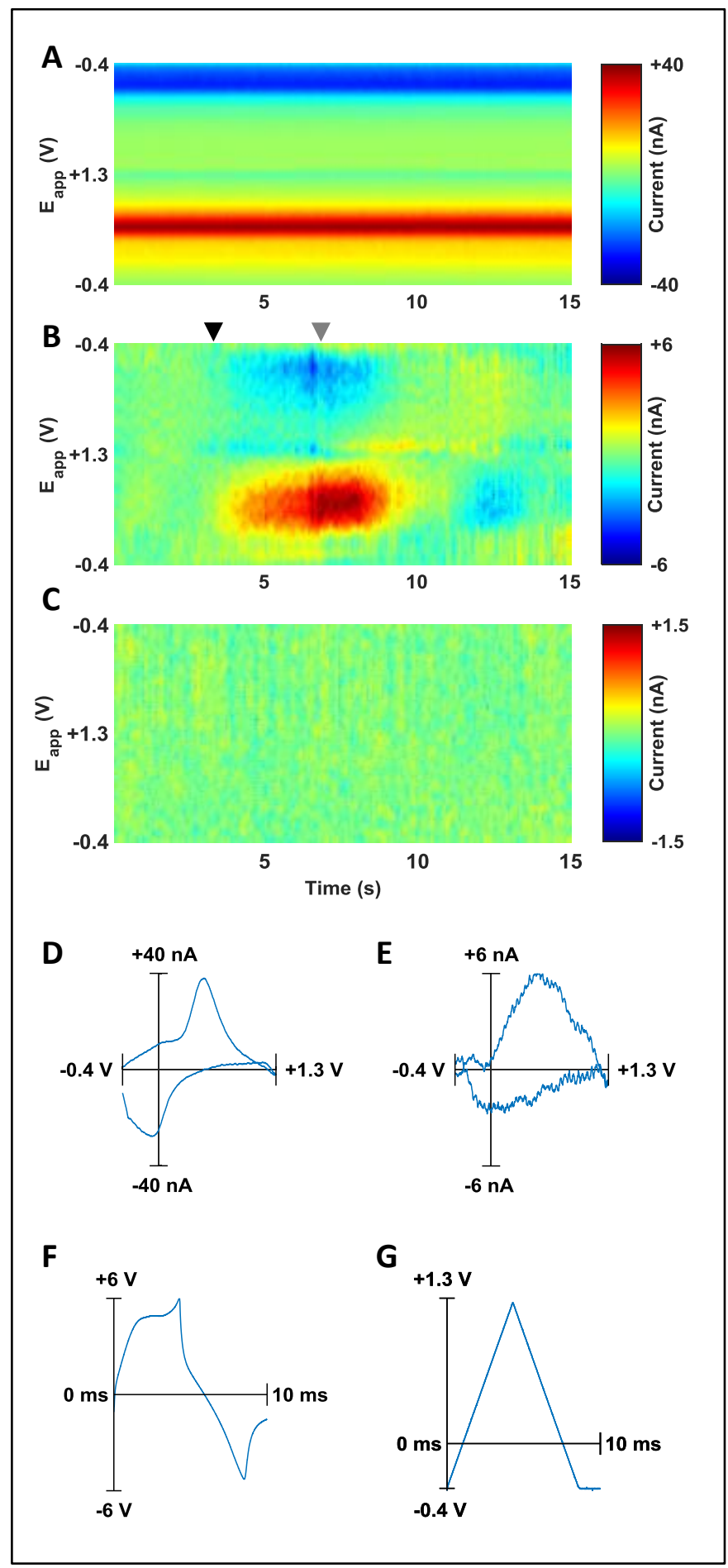

Fig. 3. Dopamine detection using the present FSCV device. (A-C) Background-subtracted voltammetric signal acquired from $1 \mu \mathrm{M}$ dopamine solution (A), the ventromedial striatum of an awake rat in response to reward 
delivery (B), and 1.2 $\mathrm{M} \Omega$ resistor simulating the working microelectrode for evaluating the noise in the FSCV device (C). (D and E) Backgroundsubtracted CVs corresponding to the plots shown in (A) and (B), respectively. (F) The signal at the output of the FSCV device for a single scan in (B). (G) The triangular waveform generated by the present FSCV device. All plots except for $(\mathrm{G})$ were filtered by centered moving averaging with a window size of 61 to suppress high frequency noise without phase shifting.

\section{DISCUSSION}

In the present work, our goal was to develop a practical, low-cost and easy-to-implement FSCV device for in vivo measurement of dopamine concentration. The solution proposed here was implemented using readily available and low-cost electronic components, and allowed measuring the changes in dopamine concentration both in vitro and in vivo (see Fig. 3). The faradaic currents caused by the oxidation and reduction of dopamine were clearly observable through the background-subtracted CVs. The present device generated smooth triangular voltammetric waveform whose timing was effectively controlled by the phase of mains line signal via the PLL circuit described here. Thanks to the present PLL circuit, we could effectively eliminate the interference caused by the mains (see Fig. 3A-C) and obtained a low-noise system. The dopamine signal could be detected in vivo without using a faraday cage or electromagnetic shielding.

The triangular waveform generator module smoothly converted pulses into monopolar triangle waves each followed by a DC holding potential. The diode in the integrator circuit effectively enabled generation of a monopolarity in the waveform. Overall, the present design approach based on use of readily available analog circuit components allowed the generation of a linear ramping signal without any discontinuity, which is unavoidably introduced by DACs, generally used for producing the voltammetric waveform in FSCV systems. In conventional systems, a DAC converter with a high resolution and update rate is preferred to improve the similarity of the generated voltammetric waveform to a triangular waveform. However, DACs with such specifications can be costly and require powerful microprocessors for control. In this work, we removed these requirements with a low-cost and practical solution. The microcontroller simply produced pulses with a specified width using timer interrupts and the waveform generator module, implemented using readily available ICs, converted these pulses into triangle waveform with adjustable holding and peak potentials. The scan rate in the present system is only limited by the gain-bandwidth product of the operational amplifiers employed.

The potentiometers involved in the triangular waveform generator module enabled setting the scan rate and waveform amplitude in a user-friendly and easy manner. It is known that there can appear a $200 \mathrm{mV}$ shift in the reference potential at the $\mathrm{Ag} / \mathrm{AgCl}$ electrodes after implantation and during longitudinal recordings [39], [40]. This shift can be identified by observing the faradaic peaks in the background currents and background-subtracted currents, and it can be compensated by offsetting the applied waveform by $+200 \mathrm{mV}$. We did not observe such shift in our in vivo test, however we believe the generated waveform can be easily offset using P3 potentiometer (Fig. 2B) if such compensation is required. When a change in the width of the rising ramp of triangular waveform is needed for different applications, the width of the pulse generated the microcontroller can be adjusted by updating its program. The width of the falling ramp can be simply controlled by potentiometer P2 (Fig. 2B) without updating the program of the microcontroller. Testing the applicability of the present waveform generator was out of the scope of this work, however we the present system can be used in the detection of other electrochemically active molecules requiring a triangular voltage scan with different holding and peak potentials [41], [42].

We optically isolated the PLL module from the waveform generator module in order to allow replacement of the microcontroller used here with other ones and prevent any noise interference which might be caused by the microcontroller operation and its clock circuitry. This design also allows replacement of the present PLL module with other simple pulse generators (e.g. function generators) when any phase-locking and elimination of mains noise is not necessary. Using such readily available signal generators, one can create the pulses with required width and frequency without using a microcontroller. Since there is optical isolation at the input of the triangular waveform generator module, the elimination of noise interference from these devices is guaranteed.

In the present system, we utilized a commutator to allow the rat freely move in the cage while it was tethered to the FSCV board. We connected the headstage batteries directly to the headstage instead of providing the supply voltage through the commutator from the FSCV board (see Fig. 1). It has been an effective solution to avoid the voltage drifts in the headstage which could appear if the headstage power were supplied through the commutator. The present system ensured stabilization of the background current within 1 hour after connecting the headstage to the implanted electrodes. Our results based on the in vivo recording (see Fig. 3B) demonstrated that the stability in background current could be effectively achieved using this configuration.

In the present system, we used a data acquisition card to record the output of the FSCV hardware with a resolution of 12-bit and a sampling rate of $200 \mathrm{KHz}$. Based on these sampling rate and resolution, we empirically found that a centered moving average filter with a windows size of 61 allowed us to visualize dopamine signal practically. We preferred using a centered moving average filter instead of a digital low-pass filter to avoid any phase shift-related distortion in the visualized background-subtracted $\mathrm{CV}$. We believe it is also possible to use a lower sampling rate (e.g. $100 \mathrm{KHz}$ ) in data acquisition while reliably detecting the faradaic currents from dopamine. The digital filter used should be updated according to the specifications of the data acquisition equipment. An analog low-pass filter can also be implemented into the amplifier module of the present device instead of performance of digital filtering. 


\section{CONCLUSION}

We described a low-cost and easy-to-implement triangular waveform generator for FSCV and validated its practicality for in vivo and in vitro detection of dopamine. We demonstrated its operation by developing a low-cost FSCV device, and interfacing the device with chronically implanted electrodes and a data acquisition system. The present device based on use of readily available analog circuit elements facilitates the implementation of a FSCV system in a standard neuroscience research laboratory. After the present system is established in a laboratory setting, the user can easily change the holding and the peak potentials of the voltammetric waveform as needed using the potentiometers included in the system. The present system generates a triangular voltammetric waveform with linear ramps in contrast to conventional systems generating the voltammetric waveform using a DAC, which technically has a limited resolution and update rate. We believe the present approach for voltammetric waveform generation can also be used in the applications targeting detection of other electrochemically active molecules requiring a triangular voltage scan.

\section{ACKNOWLEDGMENT}

This research was supported by The Scientific and Technological Research Council of Turkey (TÜBİTAK), Grant No: EEEAG-117E286 and SBAG-118S072.

\section{REFERENCES}

[1] M. Howe, P. L. Tierney, S. G. Sandberg, P. E. M. Phillips, and A. M. Graybiel, "Prolonged dopamine signalling in striatum signals proximity and value of distant rewards.," Nature, Aug. 2013, doi: 10.1038/nature12475.

[2] X. Jin and R. M. Costa, "Start/stop signals emerge in nigrostriatal circuits during sequence learning," Nature, vol. 466, no. 7305, pp. 457-462, 2010, doi: 10.1038/nature09263.

[3] J. N. Reynolds, B. I. Hyland, and J. R. Wickens, "A cellular mechanism of reward-related learning.," Nature, vol. 413, no. 6851, pp. 67-70, Sep. 2001, doi: 10.1038/35092560.

[4] W. Schultz, P. Dayan, and P. R. Montague, "A neural substrate of prediction and reward.," Science, vol. 275, no. 5306, pp. 15931599, Mar. 1997, doi: 10.1126/science.275.5306.1593.

[5] P. N. Tobler, C. D. Fiorillo, and W. Schultz, "Adaptive coding of reward value by dopamine neurons.," Science, vol. 307, no. 5715, pp. 1642-5, Mar. 2005, doi: 10.1126/science.1105370.

[6] P. E. M. Phillips, D. L. Robinson, G. D. Stuber, R. M. Carelli, and R. M. Wightman, "Real-Time Measurements of Phasic Changes in Extracellular Dopamine Concentration in Freely Moving Rats by Fast-Scan Cyclic Voltammetry," in Drugs of Abuse. Methods In Molecular Medicine, vol. 79, J. Q. Wang, Ed. New Jersey: Humana Press, 2003, pp. 443-464.

[7] D. L. Robinson, B. J. Venton, M. L. A. V Heien, and R. M. Wightman, "Detecting subsecond dopamine release with fast-scan cyclic voltammetry in vivo," Clin. Chem., vol. 49, no. 10, pp. 1763 1773, 2003, doi: 10.1373/49.10.1763.

[8] I. Willuhn et al., "Phasic Dopamine Release in the Nucleus Accumbens in Response to Pro-Social $50 \mathrm{kHz}$ Ultrasonic Vocalizations in Rats," J. Neurosci., vol. 34, no. 32, pp. 1061610623, 2014, doi: 10.1523/JNEUROSCI.1060-14.2014.

[9] M. Klanker, L. Fellinger, M. Feenstra, I. Willuhn, and D. Denys, "Regionally distinct phasic dopamine release patterns in the striatum during reversal learning," Neuroscience, no. May, 2016, doi: 10.1016/j.neuroscience.2016.05.011.

[10] M. M. Arnold, L. M. Burgeno, and P. E. M. Phillips, "Fast- Scan Cyclic Voltammetry in Behaving Animals," in Basic Electrophysiological Methods, E. Covey and M. Carter, Eds. Oxford University Press, 2015.
B. J. Venton and Q. Cao, "Fundamentals of fast-scan cyclic voltammetry for dopamine detection," Analyst, vol. 145, no. 4, pp. 1158-1168, 2020, doi: 10.1039/c9an01586h.

[12] R. M. Wightman, "Microvoltammetric Electrodes," Anal. Chem., vol. 53, no. 9, pp. 1125-1134, Aug. 1981, doi:

10.1021/ac00232a791.

[13] N. T. Rodeberg, S. G. Sandberg, J. A. Johnson, P. E. M. Phillips, and R. M. Wightman, "Hitchhiker's Guide to Voltammetry: Acute and Chronic Electrodes for in Vivo Fast-Scan Cyclic Voltammetry," ACS Chem. Neurosci., vol. 8, no. 2, pp. 221-234, Feb. 2017, doi: 10.1021/acschemneuro.6b00393.

[14] R. B. Keithley et al., "Higher sensitivity dopamine measurements with faster-scan cyclic voltammetry," Anal. Chem., vol. 83, no. 9, pp. 3563-3571, 2011, doi: 10.1021/ac200143v.

[15] R. M. Wightman, L. J. May, and A. C. Michael, "Detection of Dopamine Dynamics in the Brain," Anal. Chem., vol. 60, no. 13, pp. 769-793, Jul. 1988, doi: 10.1021/ac00164a718.

[16] M. F. Roitman, R. A. Wheeler, R. M. Wightman, and R. M. Carelli, "Real-time chemical responses in the nucleus accumbens differentiate rewarding and aversive stimuli," Nat. Neurosci., vol. 11, no. 12, pp. 1376-1377, 2008, doi: 10.1038/nn.2219.

[17] J. J. Day, M. F. Roitman, R. M. Wightman, and R. M. Carelli, "Associative learning mediates dynamic shifts in dopamine signaling in the nucleus accumbens," Nat. Neurosci., vol. 10, no. 8, pp. 1020-1028, 2007, doi: 10.1038/nn1923.

[18] M. L. a V Heien et al., "Real-time measurement of dopamine fluctuations after cocaine in the brain of behaving rats.," Proc. Natl. Acad. Sci. U. S. A., vol. 102, no. 29, pp. 10023-10028, 2005, doi: 10.1073/pnas.0504657102.

[19] P. Takmakov et al., "Carbon microelectrodes with a renewable Surface," Anal. Chem., vol. 82, no. 5, pp. 2020-2028, 2010, doi: 10.1021/ac902753x.

[20] J. O. Howell, W. G. Kuhr, R. E. Ensman, and R. Mark Wightman, "Background subtraction for rapid scan voltammetry," $J$. Electroanal. Chem. Interfacial Electrochem., vol. 209, no. 1, pp. 77-90, Sep. 1986, doi: 10.1016/0022-0728(86)80187-5.

[21] M. DeWaele et al., "A baseline drift detrending technique for fast scan cyclic voltammetry," Analyst, vol. 142, no. 22, pp. 4317-4321, 2017, doi: 10.1039/C7AN01465A.

[22] E. W. Schluter, A. R. Mitz, J. F. Cheer, and B. B. Averbeck, "Realtime dopamine measurement in awake monkeys," PLoS One, vol. 9, no. 6, 2014, doi: 10.1371/journal.pone.0098692.

[23] J. A. Johnson, N. T. Rodeberg, and R. M. Wightman, "Failure of Standard Training Sets in the Analysis of Fast-Scan Cyclic Voltammetry Data," ACS Chem. Neurosci., vol. 7, no. 3, pp. 349359, Mar. 2016, doi: 10.1021/acschemneuro.5b00302.

[24] P. Puthongkham and B. J. Venton, "Recent advances in fast-scan cyclic voltammetry," Analyst, vol. 145, no. 4, pp. 1087-1102, 2020, doi: 10.1039/c9an01925a.

[25] P. Takmakov, C. J. McKinney, R. M. Carelli, and R. M. Wightman, "Instrumentation for fast-scan cyclic voltammetry combined with electrophysiology for behavioral experiments in freely moving animals," Rev. Sci. Instrum., vol. 82, no. 7, pp. 1-6, 2011, doi: 10.1063/1.3610651.

[26] J. J. Clark, A. L. Collins, C. A. Sanford, and P. E. M. Phillips, "Dopamine encoding of Pavlovian incentive stimuli diminishes with extended training.," J. Neurosci., vol. 33, no. 8, pp. 3526-32, 2013, doi: 10.1523/JNEUROSCI.5119-12.2013.

[27] C. M. Cameron, R. M. Wightman, and R. M. Carelli, "Dynamics of rapid dopamine release in the nucleus accumbens during goaldirected behaviors for cocaine versus natural rewards," Neuropharmacology, vol. 86, no. August, pp. 319-328, 2014, doi: 10.1016/j.neuropharm.2014.08.006.

[28] N. T. Rodeberg, J. A. Johnson, C. M. Cameron, M. P. Saddoris, R. M. Carelli, and R. M. Wightman, "Construction of Training Sets for Valid Calibration of in Vivo Cyclic Voltammetric Data by Principal Component Analysis," Anal. Chem., vol. 87, no. 22, pp. 1148411491, 2015, doi: 10.1021/acs.analchem.5b03222.

[29] C. J. Kimble et al., "Wireless instantaneous neurotransmitter concentration sensing system (WINCS) for intraoperative neurochemical monitoring," in 2009 Annual International Conference of the IEEE Engineering in Medicine and Biology Society, Sep. 2009, no. September 2009, pp. 4856-4859, doi: 10.1109/IEMBS.2009.5332773.

[30] Y.-T. Li, J. R. Wickens, Y.-L. Huang, W. H. T. Pan, F.-Y. B. Chen, 
and J.-J. J. Chen, "Integrated wireless fast-scan cyclic voltammetry recording and electrical stimulation for reward-predictive learning in awake, freely moving rats.," J. Neural Eng., vol. 10, no. 4, p. 046007, 2013, doi: 10.1088/1741-2560/10/4/046007.

[31] S. D. Adams, E. H. Doeven, S. J. Tye, K. E. Bennet, M. Berk, and A. Z. Kouzani, "TinyFSCV: FSCV for the Masses," IEEE Trans. Neural Syst. Rehabil. Eng., vol. 28, no. 1, pp. 133-142, 2020, doi: 10.1109/TNSRE.2019.2956479.

[32] T. Scardochio, I. Trujillo-Pisanty, K. Conover, P. Shizgal, and P. B. S. Clarke, "The Effects of Electrical and Optical Stimulation of Midbrain Dopaminergic Neurons on Rat $50-\mathrm{kHz}$ Ultrasonic Vocalizations," Front. Behav. Neurosci., vol. 9, no. December, pp. 1-15, 2015, doi: 10.3389/fnbeh.2015.00331.

[33] A. L. Collins, V. Y. Greenfield, J. K. Bye, K. E. Linker, A. S Wang, and K. M. Wassum, "Dynamic mesolimbic dopamine signaling during action sequence learning and expectation violation," Sci. Rep., vol. 6, no. February, pp. 1-15, 2016, doi: 10.1038/srep20231.

[34] T. Claycomb et al., Analog Engineer's Circuit Cookbook: Amplifiers, 2nd ed. Texas Insturments, 2019.

[35] D. J. Michael, J. D. Joseph, M. R. Kilpatrick, E. R. Travis, and R. M. Wightman, "Improving data acquisition for fast-scan cyclic voltammetry,” Anal. Chem., vol. 71, no. 18, pp. 3941-3947, 1999, [Online]. Available: http://www.ncbi.nlm.nih.gov/entrez/query.fcgi?cmd=Retrieve \&db= PubMed\&dopt $=$ Citation\&list_uids $=10500480$.

[36] J. J. Clark et al., "Chronic microsensors for longitudinal, subsecond dopamine detection in behaving animals.," Nat. Methods, vol. 7, no. 2, pp. 126-129, 2010, doi: 10.1038/nmeth.1412.

[37] G. Paxinos and C. Watson, The rat brain in stereotaxic coordinates, 5th ed. Academic Press, 2004.

[38] L. M. O. Oliveira and D. Dimitrov, "Surgical Techniques for Chronic Implantation of Microwire Arrays in Rodents and Primates," in Methods for Neural Ensemble Recordings, 2nd ed., M. A. L. Nicolelis, Ed. Boca Raton: CRC Press, 2008.

[39] F. Moussy and D. J. Harrison, "Prevention of the Rapid Degradation of Subcutaneously Implanted Ag/AgCl Reference Electrodes Using Polymer Coatings," Anal. Chem., vol. 66, no. 5, pp. 674-679, 1994 doi: 10.1021/ac00077a015.

[40] P. Hashemi et al., "Chronically implanted, nafion-coated $\mathrm{Ag} / \mathrm{AgCl}$ reference electrodes for neurochemical applications," ACS Chem. Neurosci., vol. 2, no. 11, pp. 658-666, 2011, doi: $10.1021 / \mathrm{cn} 2000684$

[41] S. N. Berger and P. Hashemi, "Brain Chemistry: Neurotransmitters," in Reference Module in Chemistry, Molecular Sciences and Chemical Engineering, 3rd ed., Elsevier, 2018, pp. 316-331.

[42] E. S. Bucher and R. M. Wightman, "Electrochemical Analysis of Neurotransmitters," Annu. Rev. Anal. Chem., vol. 8, no. April, pp 239-261, 2015, doi: 10.1146/annurev-anchem-071114-040426. 